\title{
Multiple Recurrent Obstructive Sialolithiasis of Left Wharton's Duct - A Case Report
}

\author{
Jitender Kumar Aurora ${ }^{1}$, Shamita Tiwari ${ }^{2 *}$, Parul Tandon ${ }^{3}$, Himanshu \\ Chauhan $^{3}$, Amartya P Srivastava ${ }^{2}$ and Preeti Sharma ${ }^{4}$ \\ ${ }^{1}$ Professor and Head, Department of Oral and Maxillofacial Surgery, Saraswati \\ Dental College, Lucknow, India \\ ${ }^{2}$ Senior Lecturer, Department of Oral and Maxillofacial Surgery, Saraswati Dental \\ College, Lucknow, India \\ ${ }^{3}$ Reader, Department of Oral and Maxillofacial Surgery, Saraswati Dental College, \\ Lucknow, India \\ ${ }^{4}$ Private Practitioner, Lucknow, India
}

*Corresponding Author: Shamita Tiwari, Senior Lecturer, Department of Oral and Maxillofacial Surgery, Saraswati Dental College, Lucknow, India.
Received: September 30, 2021

Published: November 23, 2021

(C) All rights are reserved by Shamita Tiwari., et al.

\section{Abstract}

Sialolith of the salivary glands is a common finding. Superadded infections can make the condition painful. Management of sialolithiasis can be done conservatively or by intraoral surgery without recurrence. Though these can be treated successfully with rare chances of recurrence, multiple sialoliths is a rare finding. It becomes difficult for a surgeon to manage it by less invasive surgery if there are multiple recurring calculi.

This case report presents the case of a 35 year old woman who reported for the management of multiple calculi which was managed by intraoral incision and 9 sialoliths were retrieved from the Wharton's duct. Occlusal radiographs and restoration of salivary flow confirmed that all the sialoliths had been retrieved. Composition analysis was carried out for the calculi. However, recurrence occurred multiple times eventually leading to the sialadenitis of the gland. Surgical excision of the submandibular gland was performed to prevent further discomfort to the patient.

Recurrence of calculi plays a major role in deciding the method of treatment. Studies on composition analysis can help analyse the cause of multiple recurrences in future.

Keywords: Sialolith; Salivary Glands; Radiographs

\section{Introduction}

Sialolithiasis is the most common disease of salivary glands. It affects approximately 12 in 1000 of the adult population with 2:1 male predilection [1]. About $80 \%-95 \%$ of salivary calculi occur in the submandibular gland while $5 \%$ to $10 \%$ are found in the parotid gland. The least involved salivary glands are the sublingual gland and minor salivary glands with about $1 \%-2 \%$ of occurrence. The higher occurrence of sialoliths in submandibular gland may be attributed to its anatomic location, long tortuous duct with a narrow orifice compared to the main portion of duct in which saliva flows against gravity at a slower rate [2]. Salivary stones are mainly composed of calcium phosphate with smaller amounts of carbonates in the form of hydroxyapatite, smaller amounts of magnesium, potassium and traces of ammonium along with organic material mainly composed of carbohydrates and amino acids [3]. The submandibular stones consist of $82 \%$ inorganic and $18 \%$ organic material whereas parotid stones consist of $49 \%$ inorganic and $51 \%$ organic material [4]. More alkaline nature of saliva in the submandibular gland as compared to parotid gland may be a contributing factor for formation of sialoliths with high inorganic content but 
the etiology of sialolithiasis and its recurrence still remains a mystery. Clinically the calculi can be palpated as round or oval masses which can be rough or smooth and present as yellowish structures upon retrieval [5].

We present a case of multiple recurrent Wharton's duct sialoliths, treated by sialolith retrieval and recannulation thrice before excision of the submandibular salivary gland had to be performed in order to determine the etiological factors for recurrence, the role of composition of sialoliths in determining the nature of sialolithiasis and to explore possible treatment options for the same.

\section{Case Report}

A 35 year old female reported for the management of pain in the floor of the mouth which aggravated during food consumption. Intraoral palpation confirmed the presence of stones in the Wharton's duct. There was no salivary or purulent discharge from the duct. The skin over the submandibular gland was not swollen, nontender and non-erythematous. There was no lymphadenopathy. Occlusal radiograph (Figure 1) confirmed the presence of multiple small calculi in left submandibular duct. Patient also gave history of intraoral surgical stone retrieval from the same duct at some other centre four years before reporting to our centre. Her medical history was insignificant.

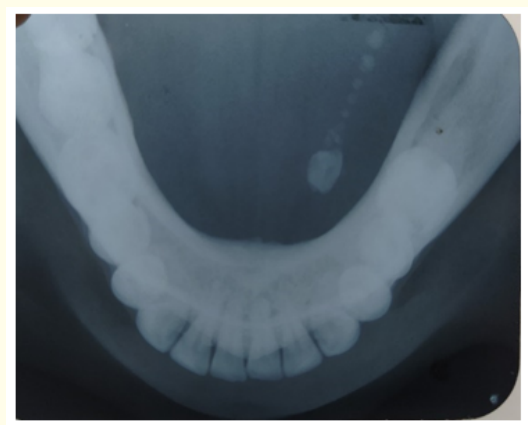

Figure 1: Occlusal radiograph showing multiple stones.

After obtaining written consent from the patient and getting all routine blood investigations done, the patient was taken up for intraoral surgery under local anaesthesia for the retrieval of calculi. Duct was tied with a suture to prevent slippage of stones posteriorly. Incision was placed over the duct exposing the sialoliths. Duct was explored and all the sialoliths were removed which was confirmed by taking occlusal radiograph. 9 calculi of variable sizes were retrieved from the duct (Figure 2). Thereafter, infants feeding (Figure 6) tube was placed inside the duct and sutured with 3-0 vicryl sutures to avoid stricture and maintain the patency of the duct (Figure 3).

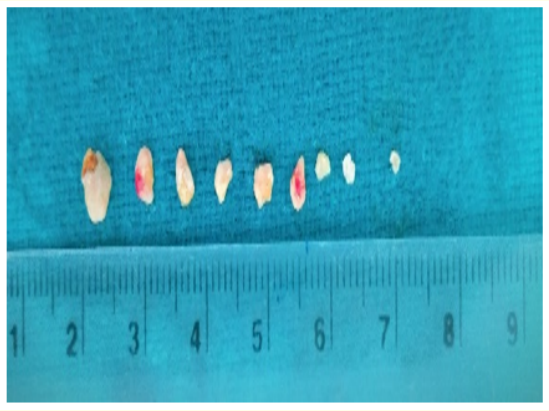

Figure 2: Retrieved calculi.

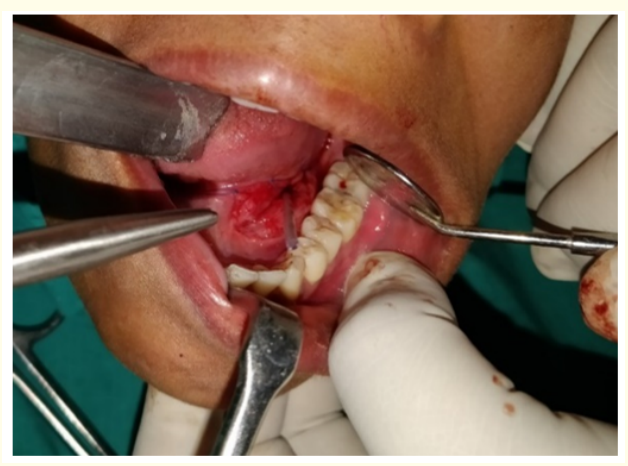

Figure 3: Placement of infant feeding tube.

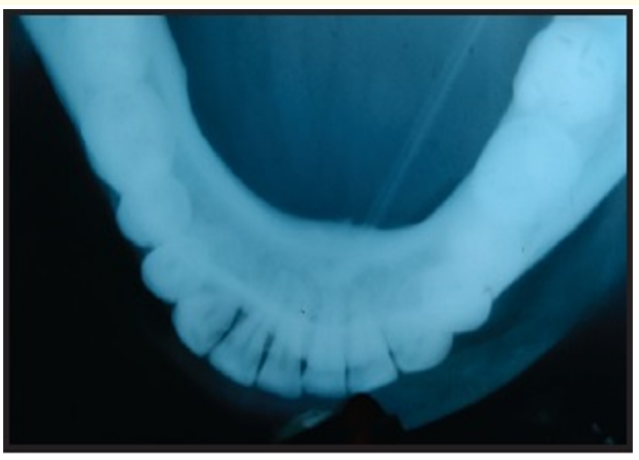

Figure 4: Final occlusal radiograph. 


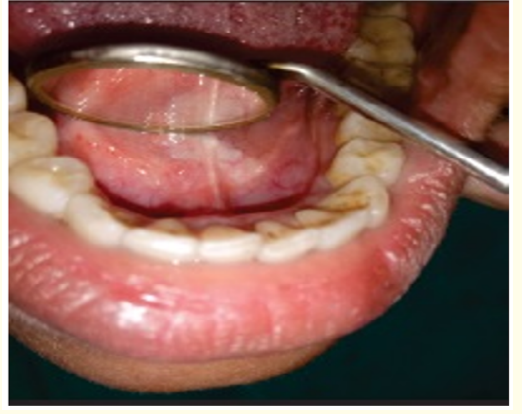

Figure 5: 2 Months post-op

The stones were referred to the laboratory for composition analysis. All the sialoliths were majorly composed of Calcium phosphate and Hydroxyapatite.

\section{Post-operative}

The patient was put on chewable Vitamin C rich diet to stimulate the flow of saliva through the duct. Ryle's tube was inserted for feeding to prevent any infection of the oral cavity. No postoperative complications were observed.

\section{Outcome and follow up}

Infant's feeding tube was taken out after 14 days and the patency of the duct was examined by monitoring the salivary flow from the duct at each follow-up. Owing to her past sialolith history, she was followed up every 3 months to check for recurrence for 1 year. Two years later, she again presented with pain and swelling in the floor of the mouth. Clinical as well as radiological examination again confirmed the presence of two small sialoliths near the opening of left Wharton's duct (Figure 7). This time she was managed conservatively by antibiotics and sialagogues which led to the expulsion of the stones. She remained asymptomatic again for the next two years after which she presented with pain, swelling and purulent discharge from the left Wharton's duct. The skin over the submandibular gland was tender and swollen this time. Occlusal Radiograph again confirmed the formation of multiple stones (Figure 8). CT scan revealed a radiodense mass measuring in the left submandibular duct with no significant dilatation (Figure 9). Surgical excision of submandibular gland using the submandibular approach along was performed under general anesthesia (Figure $10,11)$ this time. The Wharton's duct was also removed along with the gland. The wound healed uneventfully thereafter. Composition analysis of the stones retrieved from the duct revealed the presence of calcium phosphate, calcium carbonate and hydroxyapatite crystals.

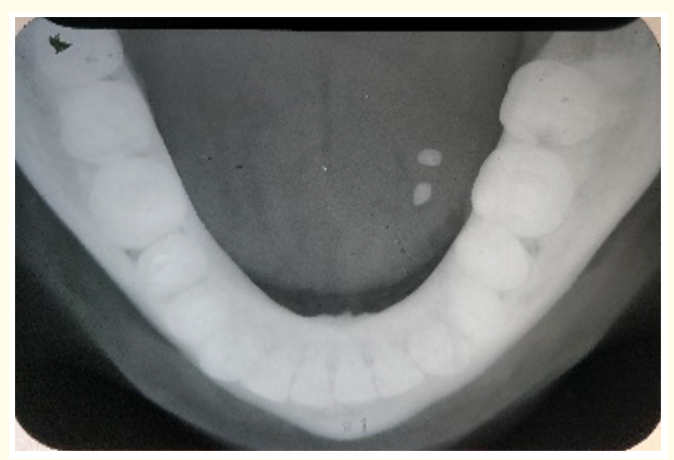

Figure 6: Occlusal radiograph taken on 2nd recurrence.

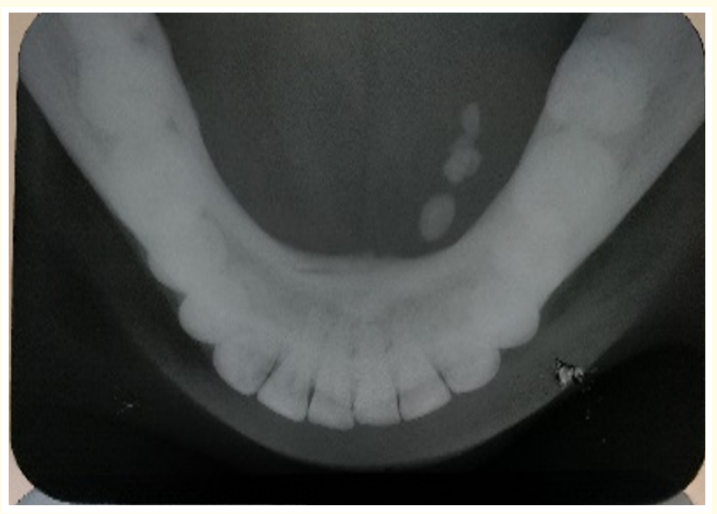

Figure 7: Occlusal Radiograph on 3rd recurrence.

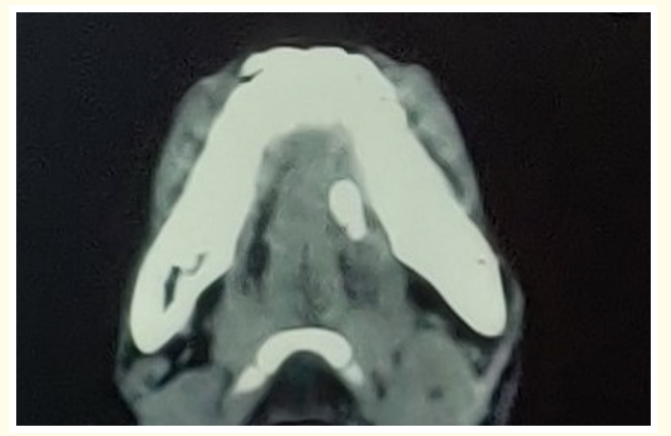

Figure 8: CECT Face revealing sialoliths in the left duct along with enlargement of left submandibular gland. 


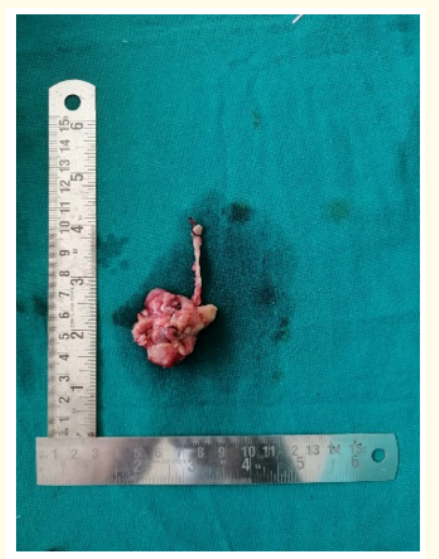

Figure 9: Submandibular gland along with the duct.

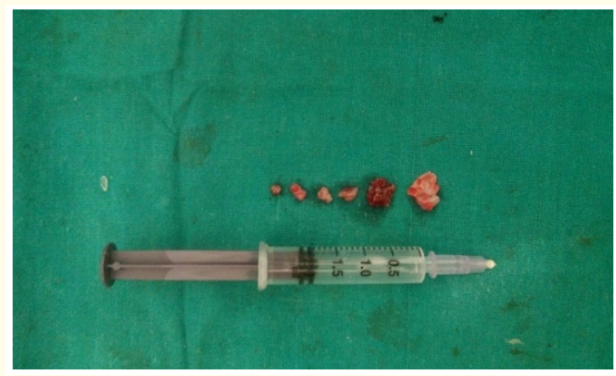

Figure 10: Stones retrieved from the duct after removal.

\section{Discussion and Conclusion}

More than one calculus in submandibular duct is a rare finding [6]. Interestingly our case had multiple recurrent sialoliths which eventually led to the excision of the gland. Management of multiple recurrent sialoliths by less invasive surgeries becomes difficult because of the stricture of the duct as well as possible infection or sialadenitis of the gland associated with multiple recurrence. The option remains to excise the gland as it happened with our case.

The management of sialoliths is decided based on the topography of the gland, the size and number of stones, the functional state of the gland, the degree of superinfection and the surgeon's technical skill. Selection of treatment mode also depends on preservation of gland function, discomfort to the patient and low level of complications [7].

Conservative approach, includes analgesia, hydration, local heat therapy, milking of the gland to expel the stone and maintain sali- vary flow. Discontinuation of anticholinergic medications can also be recommended and in case of gland superinfection antibiotics covering oral flora are suggested [1]. Gland massage after every meal with daily intake of atleast $1.5 \mathrm{~L}$ of water and sialagogues administration are also the conservative methods of management.

Invasive management of sialolithiasis consists of extracorporeal lithotripsy, sialendoscopy and surgery [8]. Sialolithiasis of submandibular gland can be managed by intraoral excision of ductal calculi with linear incision on the mucosa along the duct which makes the calculus bulge and then it is removed. While following this procedure, care must be taken not to exceed the first mandibular molar to avoid lingual nerve damage. In order to avoid its posterior migration during surgery a traction suture is placed behind the calculus which is very important for a successful surgery [9]. Extraoral approach can also be used for excision of submandibular gland and stone.

The recurrence rate of sialoliths is $1-10 \%$. Complication related to extraoral surgery is a formation of a cervical scar and also involves high risk of cranial nerves damage (VII and XII) [7].

In our case we initially believed that intraoral surgical approach to remove multiple recurrent calculi is the standard choice and recurrence may be prevented by removal of all stones also maintaining the duct patency by infant's tube insertion. But multiple recurrences in our case somehow indicate that surgical debridement of the duct is not enough to prevent recurrence. Composition of the stones may play a role in recurrence which needs to be further investigated. Further research on recurrence may help to select the correct and ideal approach to prevent recurrences.

\section{Bibliography}

1. Moghe S., et al. "Parotid sialolithiasis". BMJ Case Reports (2012).

2. Iqbal Ali., et al. "Unusually large sialolith of Wharton's duct". Annals of Maxillofacial Surgery 2.1 (2012): 70-73.

3. Williams MF. "Sialolithiasis". Otolaryngologic Clinics of North America 32.5 (1999): 819-834.

4. Bodner L. "Giant salivary gland calculi: diagnostic imaging and surgical management". Oral Surgery, Oral Medicine, Oral Pathology, Oral Radiology, and Endodontology 94.3 (2002): 320-323.

5. Arifa Sheik P., et al. "Sialolithiasis of the Submandibular Gland: Report of Cases". Cureus 11.3 (2019): e4180. 
6. Hazarika Produl., et al. "Deep and unusual sialolithiasis of submandibular duct and gland: a surgical dilemma". Indian Journal of Otolaryngology and Head and Neck Surgery: Official Publication of the Association of Otolaryngologists of India 65.4 (2013): 309-313.

7. Duong Lucas., et al. "Management of anterior submandibular sialolithiasis". Journal of Oral Medicine and Oral Surgery (2019).

8. Kraaij S., et al. "Salivary stones: symptoms, aetiology, biochemical composition and treatment". British Dental Journal 217 (2014): E23.

9. Siddiqui SJ. "Sialolithiasis: an unusually large submandibular salivary stone”. British Dental Journal 193.2 (2002): 89-91.

Volume 3 Issue 12 December 2021

(C) All rights are reserved by Shamita Tiwari., et al. 